\title{
Article \\ Minimal Optimized Effective Potentials for Density Functional Theory Studies on Excited-State Proton Dissociation
}

\author{
Pouya Partovi-Azar *(i) and Daniel Sebastiani \\ Institute of Chemistry, Martin-Luther-University Halle-Wittenberg, Von-Danckelmann-Platz 4, \\ 06120 Halle (Saale), Germany; daniel.sebastiani@chemie.uni-halle.de \\ * Correspondence: pouya.partovi-azar@chemie.uni-halle.de
}

\begin{abstract}
Recently, a new method [P. Partovi-Azar and D. Sebastiani, J. Chem. Phys. 152, 064101 (2020)] was proposed to increase the efficiency of proton transfer energy calculations in density functional theory by using the $T_{1}$ state with additional optimized effective potentials instead of calculations at $S_{1}$. In this work, we focus on proton transfer from six prototypical photoacids to neighboring water molecules and show that the reference proton dissociation curves obtained at $S_{1}$ states using time-dependent density functional theory can be reproduced with a reasonable accuracy by performing $\mathrm{T}_{1}$ calculations at density functional theory level with only one additional effective potential for the acidic hydrogens. We also find that the extra effective potentials for the acidic hydrogens neither change the nature of the $\mathrm{T}_{1}$ state nor the structural properties of solvent molecules upon transfer from the acids. The presented method is not only beneficial for theoretical studies on excited state proton transfer, but we believe that it would also be useful for studying other excited state photochemical reactions.
\end{abstract}

Keywords: density functional theory; time-dependent density functional theory; optimized effective potentials; excited states; proton dissociation

Citation: Partovi-Azar, P.

Sebastiani, D. Minimal Optimized Effective Potentials for Density Functional Theory Studies on Excited-State Proton Dissociation. Micromachines 2021, 12, 679. https://doi.org/10.3390/mi12060679

Academic Editor: István Rajta

Received: 7 May 2021

Accepted: 9 June 2021

Published: 10 June 2021

Publisher's Note: MDPI stays neutral with regard to jurisdictional claims in published maps and institutional affiliations.

Copyright: (c) 2021 by the authors. Licensee MDPI, Basel, Switzerland. This article is an open access article distributed under the terms and conditions of the Creative Commons Attribution (CC BY) license (https:// creativecommons.org/licenses/by/ $4.0 /)$.

\section{Introduction}

Many fundamental chemical processes are triggered when the electrons in a system are excited. The interaction of a molecule with its environment at an electronic excited state can be largely different from that in the electronic ground state. Therefore, it has become possible to indirectly investigate the nature of such interactions as well as structural properties of solvents by studying the change in properties of molecular probes upon excitation [1-5]. Electronic excitations can also lead to inter- or intramolecular proton transfers [6-11]. In particular, photoinduced proton transfer in solutions is of fundamental interest in a large variety of chemical and biological applications such as energy storage systems and sensors [12-18].

From a theoretical standpoint, ab initio molecular dynamics simulation (AIMD) is a suitable tool for studying local structure as well as structural dynamics of solute and solvent molecules, taking both electronic structure and thermal effects into account [19]. Density functional theory (DFT) $[20,21]$ is usually the method of choice for including electronic degrees of freedom in AIMD simulations at ground state, due to a reasonable balance between accuracy and computational efficiency. However, electronic excited states are not accessible in DFT and higher-level methods, such as time-dependent DFT (TDDFT) [22], equation of motion coupled cluster [23-25], etc., are needed. Nevertheless, force calculations using higher-level methods are prohibitively time consuming, making AIMD simulations based on these methods limited only to small system sizes and short simulation times.

In the past years, there have been various approaches to improve DFT description of proton transfer at excited states [26-33]. Recently, a method has been proposed where $\mathrm{T}_{1}$ state is used and further modified using effective potentials for acidic atoms to mimic the 
actual $S_{1}$ state [34]. $T_{1}$ state, unlike $S_{1}$, is variationally accessible in DFT and therefore the proposed method allows for efficient calculation of proton transfer energies and barriers at excited state. The modification is done by adding effective potentials, which are represented via atom-centered Gaussian functions. It has been demonstrated that the proton transfer energy can be accurately obtained by optimizing one additional effective potential per nonhydrogen atoms in a photoacid. It has also been shown that in order to obtain the correct kinetics of the proton dissociation reaction, one would need to optimize an additional effective potential for the acidic hydrogen as well. Here, we explore the possibility of finding the whole dissociation curve by optimizing only one additional non-local effective potential, represented as an expansion in terms of Gaussian projectors and only for the acidic hydrogens. As target photoacids, we consider phenol, 2- and 4-cyanophenol, 1- and 2-naphthol, and 7-hydroxyquinoline.

We emphasize that in this work, the aim is solely to reproduce the proton dissociation curves of the above photoacids at their respective first excited state. Although our preliminary investigations indicate that correct excited state wavefunctions along the reaction path could, in principle, be obtained using additional effective potentials, this still needs to be studied in detail and goes beyond the scope of the present article.

\section{Methodology}

The reference calculations are performed at TDDFT level using the Orca code $[35,36]$. Application-oriented calculations are performed at the DFT level along with pseudopotential approximation using the CP2K software $[37,38]$. All-electron DFT and TDDFT calculations with Orca are carried out using the correlation-corrected cc-pVTZ basis set [39] together with $\omega$ B97X-D3 range-separated, hybrid exchange-correlation (XC) functional [40]. The $\omega$ B97X-D3 functional has been already demonstrated to produce excited-state properties, including proton dissociation curves, with very good agreement with higher-level quantum chemical methods [34]. Therefore, in this work the reference calculations are carried out using $\omega$ B97X-D3 functional at TDDFT level. In the TDDFT calculations, the Tamm-Dancoff approximation [41,42] is used, and in all excited state calculations the target electronic state is set to $S_{1}$ while, altogether, 10 excited state roots are computed.

The proton transfer energy is defined based on dissociation of a proton from prototypical photoacids phenol, 2- and 4-cyanophenol, 1- and 2-naphthol, and 7-hydroxyquinoline (7HQ), (Figure 1a,b) to one neighboring water molecule.

The ground state structures of these complexes are first optimized in vacuum using all-electron DFT calculations with $\omega$ B97X-D3 XC functional. Afterwards, the hydrogen is gradually moved from each photoacid along the $\mathrm{O}-\mathrm{H} \cdots \mathrm{O}$ line toward the water molecule with $0.1 \AA$ strides, and the corresponding total energies are calculated (see Figure 1c,d for 1-naphthol).

As for the additional effective potentials for the acidic hydrogens, here we choose Goedecker-Teter-Hutter (GTH)-type Gaussian functions [43,44]. The analytic form of these potentials provides considerable efficiency in numerical calculations using plane-wave and mixed Gaussian/plane-wave basis sets [45-47]. Our additional effective potential is expressed using the non-local potential,

$$
\begin{aligned}
& V_{\mathrm{nl}}\left(\mathbf{r}, \mathbf{r}^{\prime}\right)=\sum_{l m} \sum_{i j}\left\langle\mathbf{r} \mid p_{i}^{l m}\right\rangle h_{i j}^{l}\left\langle p_{j}^{l m} \mid \mathbf{r}^{\prime}\right\rangle \\
& \left\langle\mathbf{r} \mid p_{i}^{l m}\right\rangle=N_{i}^{l} Y^{l m}(\theta, \phi) r^{l+2 i-2} \exp \left[-\frac{1}{2}\left(\frac{r}{r_{l}}\right)^{2}\right] .
\end{aligned}
$$

Here, $\left\langle\mathbf{r} \mid p_{i}^{l m}\right\rangle$ are Gaussian-type projectors and $h_{i j}^{l m}$ denote the $i \times j$ symmetric coefficient matrix for the angular momentum channel $l$, while $r_{l}$ represents the corresponding effective radius of the projector. $Y^{l m}(\theta, \phi)$ are the spherical harmonics and $N_{i}^{l}$ are normalization constants. We find that appropriate effective potentials for the acidic hydrogens have to be effective only at typical $\mathrm{OH}$ bond distances (covalent and H-bonded, i.e., $\sim 1 \AA$ ) 
and longer, but not in the region of the nucleus itself. In Equation (1), we consider $i=1,2$ and in order to be able to impose the above condition, we set $h_{11}^{0}$ explicitly to zero and optimize three parameters, i.e., $h_{12}^{0}$ and $h_{22}^{0}$ together with $r_{0}$.

(a)<smiles>CCOc1c(C)c(C)c(C)c(C)c1C</smiles>

Phenol

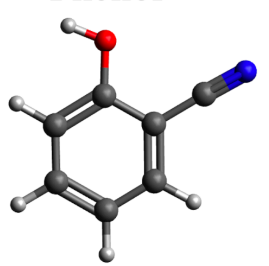

2-cyanophenol<smiles>C=Cc1c(C)c(C)c(C)c(C)c1C</smiles>

4-cyanophenol (b)

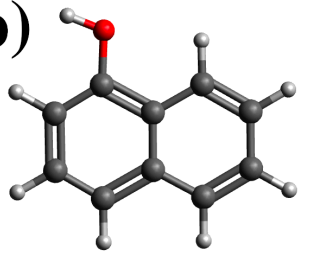

1-naphthol

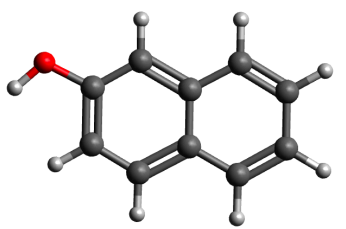

2-naphthol

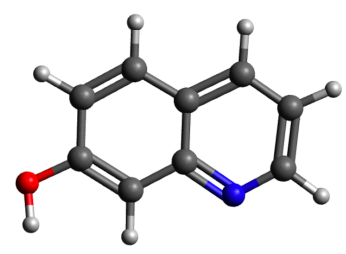

7-hydroxyquinoline (c)
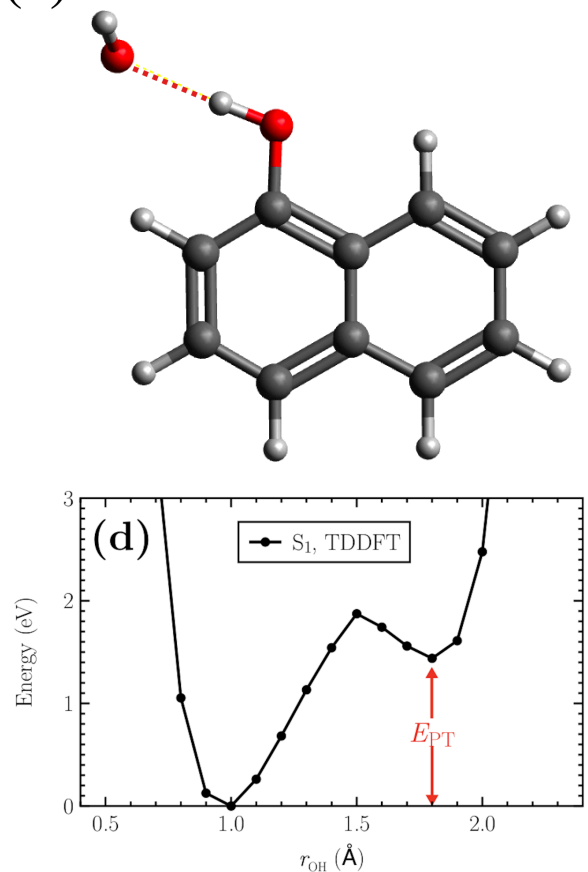

Figure 1. (a,b) The prototypical photoacids considered in this work. (c) 1-Naphthol/water complex. Similar complexes are used for each photoacid in order to calculate the respective proton dissociation curves. (d) Reference proton dissociation curve for the 1-naphthol/water complex in (c) obtained using TDDFT calculations at $\mathrm{S}_{1}$ state.

By minimizing the function

$$
f\left(r_{0}, \mathbf{h}^{0}\right)=\left[E_{\text {ref }}^{\mathrm{S}_{1}}-E_{\mathrm{DFT}}^{\mathrm{T}_{1}}\left(r_{0}, \mathbf{h}^{0}\right)\right]^{2}
$$

we require that DFT values for the proton transfer energies calculated at the $T_{1}$ state with additional effective potentials to be as close as possible to those obtained at the $S_{1}$ state of the target complexes using TDDFT. Here, $E$ denotes the proton transfer energy, $\mathbf{h}^{0}$ is a vector with components representing projector coefficients $h_{12}^{0}$ and $h_{22}^{0}$ for the acidic hydrogen. $T_{1}$ state is chosen as the starting electronic configuration because the previous studies have shown that it can partially capture the excited state properties of similar photoacids [10]. The minimization of the function $f$ is done using gradient descent method, where the step size is updated every five steps using the Barzilai-Borwein method [48]. The starting value for the projector coefficients $h_{12}^{0}$ and $h_{22}^{0}$ is set to zero, while for $r_{0}$, we use $1.0 \AA$ as a starting value. Additionally, we use the BLYP XC functional $[49,50]$ in the DFT calculations along with Grimme's dispersion correction [51] and a triple- $\zeta$ TZVP-MOLOPT basis set [38]. For dipole moment calculations at the $\mathrm{T}_{1}$ state with the extra optimized effective potentials, we use maximally localized Wannier functions [52].

\section{Results and Discussion}

Natural transition orbitals [53] show that in all the photoacids considered here, the main contribution (larger than $70 \%$ in all cases) to the $S_{0} \rightarrow S_{1}$ and $S_{0} \rightarrow T_{1}$ excitations comes from a $\mathrm{HOMO}$ to LUMO transition with a $\pi-\pi^{*}$ nature. As an example, the $\mathrm{HOMO}$ and LUMO orbitals of 7HQ at its ground state are shown in Figure 2. 
(a)

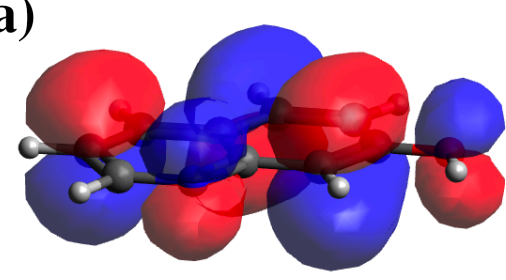

(b)

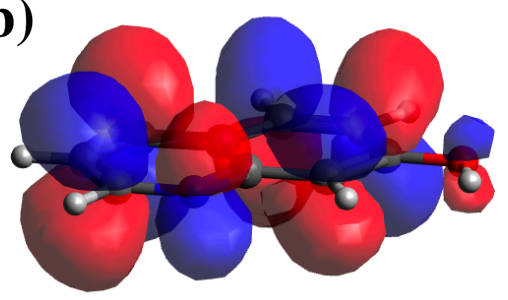

Figure 2. (a) HOMO and (b) LUMO orbitals of 7HQ photoacid at ground state calculated at DFT level.

We also observe that both excitations result in a depletion of charge around the acidic hydrogen.

We find that a better agreement with the reference TDDFT calculations can be reached by adapting the following optimization procedure: (i) we optimize $r_{0}$ and $h_{12}^{0}$ to obtain the correct proton transfer energies; (ii) using these optimized parameters, we optimize $h_{22}^{0}$ and again $r_{0}$ to reach the correct values for the transition energies. This procedure can be iterated until a convergence is reached. Based on our findings, typically two iterations are enough to reach the converged values.

The optimized values of $r_{0}, h_{12}^{0}$, and $h_{22}^{0}$ for the photoacids considered in this work are given in Table 1.

Table 1. Parameters for the acidic hydrogens (in atomic units) optimized for excited state proton transfer calculations.

\begin{tabular}{cccc}
\hline Phenol-Based Photoacids & & & \\
\hline & $r_{0}$ (a.u.) & $h_{12}^{0}$ & $h_{22}^{0}$ \\
\hline Phenol & 2.112996 & 0.465280 & 0.460871 \\
2-Cyanophenol & 1.797464 & 0.094554 & 0.395386 \\
4-Cyanophenol & 2.002620 & 0.176501 & 0.479233 \\
\hline Naphthol-Based Photoacids & & & \\
\hline & $r_{0}$ (a.u.) & $h_{12}^{0}$ & $h_{22}^{0}$ \\
\hline 1-Naphthol & 2.019293 & 0.333423 & 0.4976893 \\
2-Naphthol & 1.979366 & 0.269279 & 0.3800635 \\
7HQ & 2.085884 & 0.132695 & 0.3663171 \\
\hline
\end{tabular}

We also observe that the parameter values only marginally differ from the values reported in Table 1 when another XC functional is used for DFT calculations at $\mathrm{T}_{1}$ state. The difference is found to be less than $5 \%$ for the case of PBE functional [54]. Proton dissociation curves calculated using the parameters in Table 1 are shown in Figure $3 a, b$ for phenol- and naphthol-based photoacids, respectively.

The reference energy calculations using TDDFT at respective $S_{1}$ states are denoted with crosses. The dissociation curves at the $\mathrm{T}_{1}$ state with the optimized potentials ( $\mathrm{T}_{1} / \mathrm{opt}$.) are obtained after repeating the optimization steps (i) and (ii) for two iterations. Gray dashed lines show the dissociation curves obtained at respective $T_{1}$ states without any additional potentials. The $S_{1}$ TDDFT energies during the proton dissociation are well reproduced by DFT calculations at $\mathrm{T}_{1}$ state with the optimized parameters giving a mean absolute error (MAE) of $\sim 204$ and $\sim 255 \mathrm{meV}$ in 0.8 to $2.0 \AA \mathrm{O} \cdots \mathrm{H}$ distances for phenoland naphthol-based photoacids, respectively. Restricting the error estimation only to the two minima and the transition point, MAE is calculated to be $\sim 55$ and $\sim 78 \mathrm{meV}$ for phenoland naphthol-based photoacids, respectively. However, the $\mathrm{OH}$ covalent bond distance in the photoacids is underestimated in almost all cases (except for 7HQ) by, at most, $0.1 \AA$. The $\mathrm{O} \cdots \mathrm{H}$ distance at the transition state is correctly reproduced for 4-cyanophenol and 1-naphthol, while it is underestimated for 2-naphthol, 7HQ, and 2-cyanophenol. It is 
slightly overestimated for phenol. Additionally, we find that the optimized potentials do not alter the nature of the $\mathrm{T}_{1}$ states. In the case of $7 \mathrm{HQ}$, for example, the maximum change in the atomic orbital coefficients contributing to the HOMO molecular orbital of majority spin obtained using $\mathrm{T}_{1} /$ opt. and that computed at $\mathrm{T}_{1}$ state without any additional potential is found to be less than $10 \%$. Therefore, the shape of these orbitals are almost identical in $\mathrm{T}_{1}$ and $\mathrm{T}_{1}$ /opt. calculations. However, we observe a slight change in the HOMO-1 orbital in $\mathrm{T}_{1}$ and $\mathrm{T}_{1}$ /opt. calculations. Our specific inspection shows that in the case of $7 \mathrm{HQ}$, it is mainly caused by a $\sim 63 \%$ change in the $s$ atomic orbital coefficient of the oxygen atom in the HOMO- 1 wavefunction at $\mathrm{T}_{1}$ /opt. calculations.

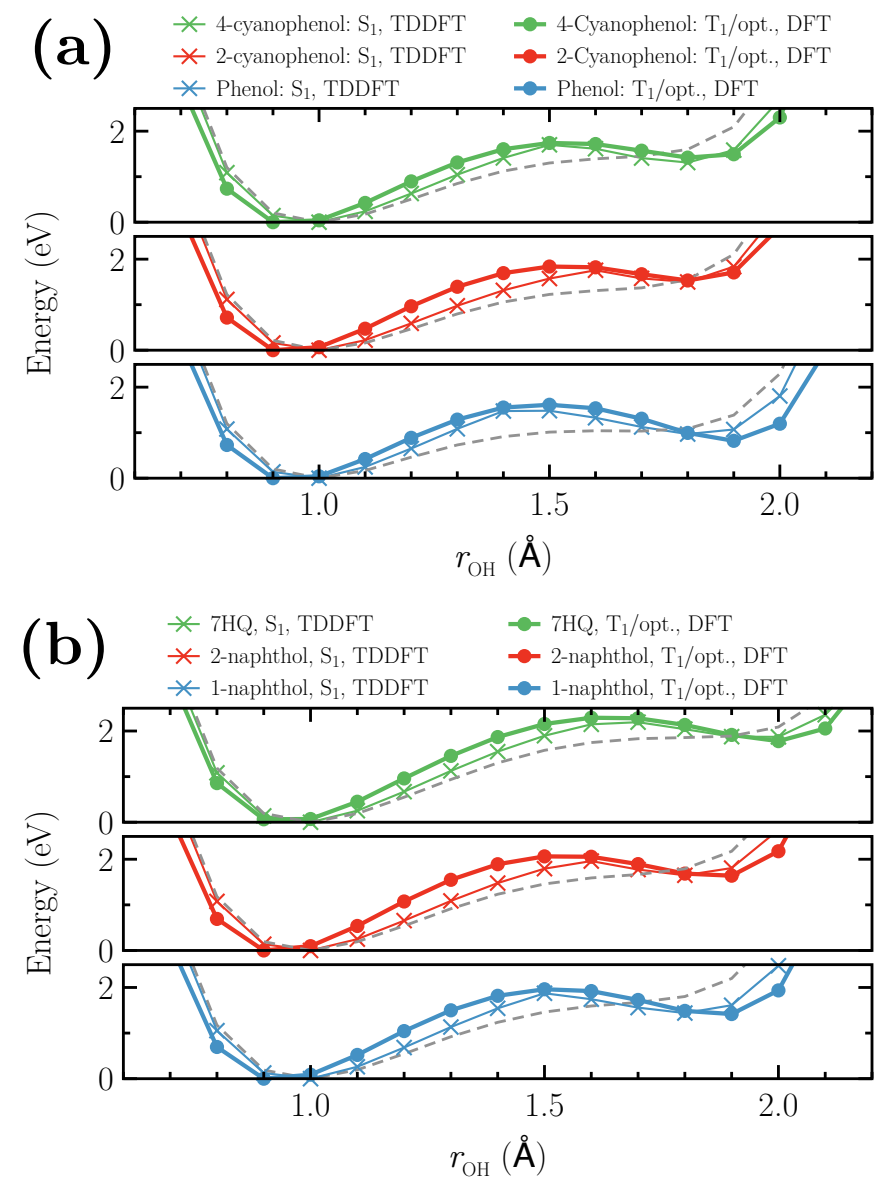

Figure 3. Proton dissociation curves for (a) phenol- and (b) naphthol-based photoacids. The reference data points obtained using TDDFT at $\mathrm{S}_{1}$ states are shown as crosses. DFT results at $\mathrm{T}_{1}$ state with the additional optimized effective potentials for the acidic hydrogens are shown as solid circles. Gray dashed lines show the dissociation curves obtained at $\mathrm{T}_{1}$ states without any additional potentials.

Additionally, to assess the performance of the optimized effective potentials for the acidic hydrogens, we calculate the molecular dipole moments as a function of $r_{\mathrm{OH}}$ around the respective global minimum of the proton dissociation curve up to the corresponding transition distance. The calculated dipole moments are presented in Figure $4 \mathrm{a}, \mathrm{b}$. The lower panels in Figure 4 show the length of the dipole moment vectors while the upper panels present the angle between the dipole moment vectors calculated at $T_{1}$ state together with the optimized effective potentials for the acidic hydrogens and the ones computed at $S_{1}$ state for the same systems using TDDFT. The reference dipole moment lengths are shown in black crosses and the gray dashed lines represent the results obtained at the $\mathrm{T}_{1}$ state without any optimized potentials. Both the length and the direction of the dipole moments obtained in the $\mathrm{T}_{1}$ /opt. calculations are in a very good agreement with the reference dipole moments in phenol- as well as naphthol-based photoacids in the $\mathrm{O} \cdots \mathrm{H}$ distances shown 
in Figure $4 \mathrm{a}, \mathrm{b}$. However, for longer $\mathrm{O} \cdots \mathrm{H}$ distances, the $\mathrm{T}_{1}$ /opt. results become largely different from the reference data due to non-adiabatic level crossing from $S_{1}$ to $S_{2}$, which was found to occur in all the systems considered in this work. The eigenvalue of the $S^{2}$ operator, i.e., 2 , in both calculations remains virtually identical in the $\mathrm{O} \cdots \mathrm{H}$ distances shown in Figure 4.

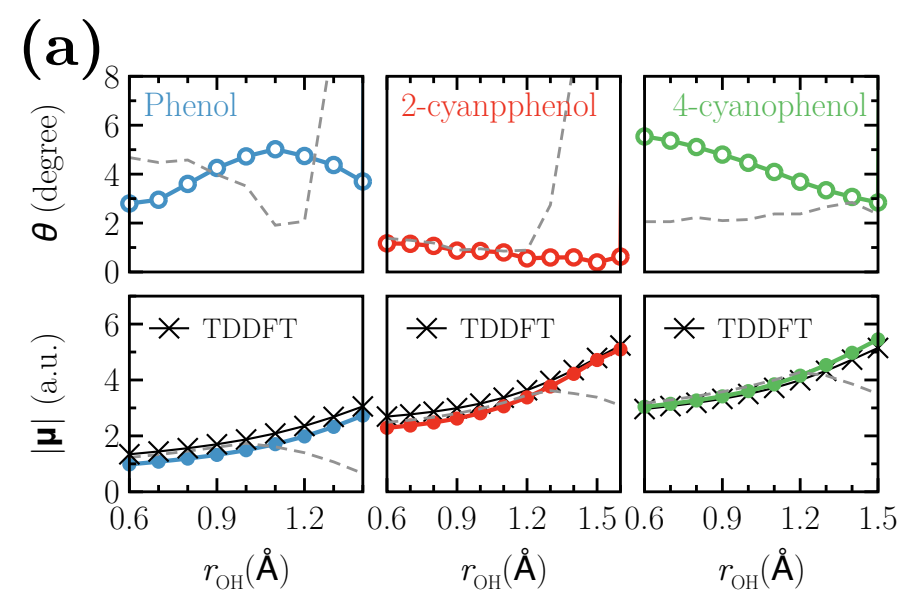

(b)

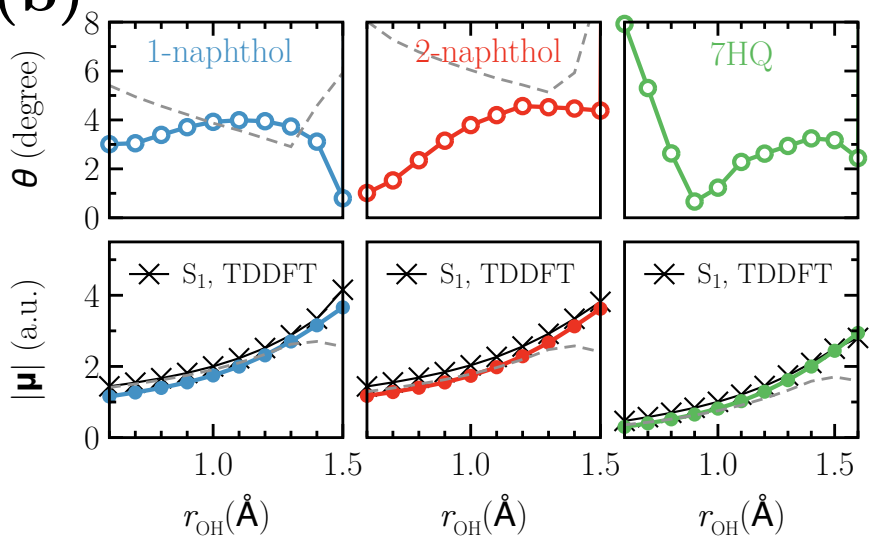

Figure 4. Dipole moments of (a) phenol- and (b) naphthol-based photoacids. The lower panels show the length of the dipole moment vector, while the upper panels present the angle between the dipole moment vectors calculated at $\mathrm{T}_{1}$ state together with the optimized effective potentials and the ones computed at $\mathrm{S}_{1}$ state for the same systems using TDDFT. The black crosses in the lower panels denote the reference TDDFT values. Gray dashed curves represent the values obtained at $\mathrm{T}_{1}$ state without any additional potential.

Finally, we focus on a situation in which the acidic proton has transferred to the neighboring water molecule, for example, in an AIMD simulation. The aim here is to make sure that the covalent bonding to the water molecule is not changed upon transfer of the proton from the photoacids. To this end, we perform a series of geometry optimizations on a hydronium cation with one of the hydrogens carrying the extra optimized potentials (number 3 in Figure 5). The geometrical properties of such a hydronium cation are given in Table 2. The bond lengths and angles are observed to remain very close to the ones obtained for a hydronium cation without any additional potential. The biggest difference is found for phenol in terms of the angles between the bonds. This finding indicates that the effective potentials for the acidic hydrogens can not only be used to reproduce the correct energies and barriers of excited state proton transfer reactions from a photoacid to a solvent molecules but can also be utilized throughout an AIMD simulation without the need to switch it off for the solvent molecules, which usually remain at their ground electronic state. 


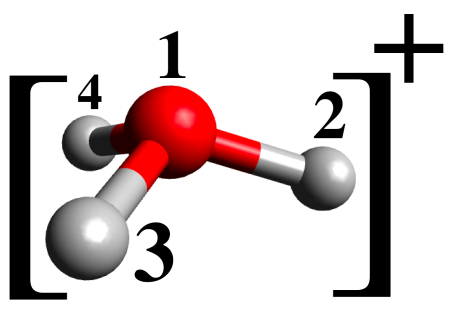

Figure 5. A hydronium cation which is assumed to form after proton transfer from the photoacids considered in this work. The transferred proton is denoted as 3.

Table 2. Bond lengths and angles of a hydronium molecule with additional optimized effective potential on one proton (number 3 in Figure 5). The optimized parameters are the same as in Table 1.

\begin{tabular}{lcccccc}
\hline From & $\mathbf{d}_{\mathbf{1 2}}(\mathbf{\AA})$ & $\mathbf{d}_{\mathbf{1 3}}(\mathbf{\AA})$ & $\mathbf{d}_{\mathbf{1 4}}(\AA)$ & $\angle \mathbf{2 1 3}\left(^{\circ}\right)$ & $\angle \mathbf{2 1 4}\left(^{\circ}\right)$ & $\left.\angle \mathbf{3 1 4} \mathbf{(}^{\circ}\right)$ \\
\hline Phenol & 0.99 & 0.97 & 0.99 & 106 & 106 & 106 \\
2-cyanophenol & 0.99 & 0.97 & 0.99 & 110 & 109 & 110 \\
4-cyanophenol & 0.99 & 0.97 & 0.99 & 108 & 108 & 108 \\
\hline 1-naphthol & 0.99 & 0.97 & 0.99 & 108 & 107 & 108 \\
2-naphthol & 0.99 & 0.97 & 0.99 & 109 & 108 & 109 \\
7HQ & 0.99 & 0.97 & 0.99 & 109 & 109 & 109 \\
\hline No OEP & 0.99 & 0.99 & 0.99 & 111 & 111 & 111 \\
\hline
\end{tabular}

\section{Conclusions}

In this work, we have proposed and optimized a specific form of effective potentials for acidic hydrogens to improve the efficiency of density functional theory calculations in predicting energies and barriers of proton transfer reactions at excited state. In this approach, a non-local potential of Goedecker-Teter-Hutter type, represented as an expansion in terms of Gaussian projectors, is used for acidic hydrogens in order to reconstruct the correct proton dissociation curves at $S_{1}$ state by performing $T_{1}$ calculations at density functional theory level. We have employed this approach to study proton dissociation from several prototypical photoacids to neighboring water molecules. We have shown that after optimizing the additional effective potentials for the acidic hydrogens, both reaction energies and barriers of the proton dissociation reactions can be reproduced with reasonable accuracy. Additionally, we have found that, in all cases considered here, the structural properties of the hydronium cation formed after the full transfer of the acidic hydrogens to the neighboring molecule is nearly identical to the one without additional potentials. This implies that the additional effective potentials can be utilized for the whole system, i.e. both photoacid and solvent molecules, without the need to switching them off after the proton transfer from the excited photoacid to the solvent. The presented method is expected to be useful for studying excited state photochemical reactions in general systems.

Author Contributions: Conceptualization, P.P.-A. and D.S.; methodology, P.P.-A. and D.S.; software, P.P.-A.; validation, P.P.-A.; formal analysis, P.P.-A.; investigation, P.P.-A.; resources, P.P.-A. and D.S.; data curation, P.P.-A.; writing-original draft preparation, P.P.-A.; writing-review and editing, P.P.-A. and D.S.; visualization, P.P.-A.; supervision, P.P.-A.; project administration, P.P.-A. and D.S.; funding acquisition, P.P.-A. and D.S. All authors have read and agreed to the published version of the manuscript.

Funding: This research was funded by DFG grants number PA3141/3-1 (Project number 420536636) and PA3141/5-1 (Project number 446879138).

Acknowledgments: The author gratefully acknowledges DFG funding via projects PA3141/3-1 (Project number 420536636) and PA3141/5-1 (Project number 446879138). The computations have been mostly performed on a Bull Cluster at the Center for Information Services and High Performance 
Computing (ZIH) at TU Dresden via the project 'p_oligothiophenes'. The authors also thank the HPC Service of ZEDAT, Freie Universität Berlin, for computing time.

Conflicts of Interest: The authors declare no conflict of interest.

\section{References}

1. Horng, M.; Gardecki, J.; Papazyan, A.; Maroncelli, M. Subpicosecond measurements of polar solvation dynamics: Coumarin 153 revisited. J. Phys. Chem. 1995, 99, 17311-17337. [CrossRef]

2. Haran, G.; Sun, W.D.; Wynne, K.; Hochstrasser, R.M. Femtosecond far-infrared pump-probe spectroscopy: A new tool for studying low-frequency vibrational dynamics in molecular condensed phases. Chem. Phys. Lett. 1997, 274, 365-371. [CrossRef]

3. Dallmann, A.; Pfaffe, M.; Mügge, C.; Mahrwald, R.; Kovalenko, S.A.; Ernsting, N.P. Local THz time domain spectroscopy of duplex DNA via fluorescence of an embedded probe. J. Phys. Chem. B 2009, 113, 15619-15628. [CrossRef]

4. Pérez Lustres, J.L.; Kovalenko, S.A.; Mosquera, M.; Senyushkina, T.; Flasche, W.; Ernsting, N.P. Ultrafast Solvation of N-Methyl-6quinolone Probes Local IR Spectrum. Angew. Chem. Int. Ed. 2005, 44, 5635-5639. [CrossRef]

5. Pérez-Lustres, J.; Rodriguez-Prieto, F.; Mosquera, M.; Senyushkina, T.; Ernsting, N.; Kovalenko, S. Ultrafast proton transfer to solvent: Molecularity and intermediates from solvation-and diffusion-controlled regimes. J. Am. Chem. Soc. 2007, 129, 5408-5418. [CrossRef] [PubMed]

6. Syage, J.; Steadman, J. Picosecond measurements of phenol excited-state proton transfer in clusters. I. Solvent basicity and cluster size effects. J. Chem. Phys. 1991, 95, 2497-2510. [CrossRef]

7. Arnaut, L.G.; Formosinho, S.J. Excited-state proton transfer reactions I. Fundamentals and intermolecular reactions. J. Photochem. Photobiol. A Chem. 1993, 75, 1-20. [CrossRef]

8. Formosinho, S.J.; Arnaut, L.G. Excited-state proton transfer reactions II. Intramolecular reactions. J. Photochem. Photobiol. A Chem. 1993, 75, 21-48. [CrossRef]

9. Allolio, C.; Sebastiani, D. Approaches to the solvation of the molecular probe N-methyl-6-quinolone in its excited state. Phys. Chem. Chem. Phys. 2011, 13, 16395-16403. [CrossRef]

10. Bekçioğlu, G.; Hoffmann, F.; Sebastiani, D. Solvation-dependent latency of photoacid dissociation and transient IR signatures of protonation dynamics. J. Phys. Chem. A 2015, 119, 9244-9251. [CrossRef]

11. Georgiev, A.; Antonov, L. 8-(Pyridin-2-yl) quinolin-7-ol as a Platform for Conjugated Proton Cranes: A DFT Structural Design. Micromachines 2020, 11, 901. [CrossRef] [PubMed]

12. Bort, G.; Gallavardin, T.; Ogden, D.; Dalko, P.I. From one-photon to two-photon probes: "Caged" compounds, actuators, and photoswitches. Angew. Chem. Int. Ed. 2013, 52, 4526-4537. [CrossRef]

13. Bucher, D.B.; Schlueter, A.; Carell, T.; Zinth, W. Watson-Crick Base Pairing Controls Excited-State Decay in Natural DNA. Angew. Chem. Int. Ed. 2014, 53, 11366-11369. [CrossRef]

14. Conrad, K.S.; Manahan, C.C.; Crane, B.R. Photochemistry of flavoprotein light sensors. Nat. Chem. Biol. 2014, 10, 801-809. [CrossRef]

15. Morrone, J.A.; Tuckerman, M.E. Ab initio molecular dynamics study of proton mobility in liquid methanol. J. Chem. Phys. 2002, 117, 4403-4413. [CrossRef]

16. Kermis, H.R.; Kostov, Y.; Harms, P.; Rao, G. Dual excitation ratiometric fluorescent pH sensor for noninvasive bioprocess monitoring: development and application. Biotechnol. Prog. 2002, 18, 1047-1053. [CrossRef] [PubMed]

17. Izvekov, S.; Voth, G.A. Ab initio molecular-dynamics simulation of aqueous proton solvation and transport revisited. J. Chem. Phys. 2005, 123, 044505. [CrossRef]

18. Geissler, P.L.; Dellago, C.; Chandler, D.; Hutter, J.; Parrinello, M. Ab initio analysis of proton transfer dynamics in $\left(\mathrm{H}_{2} \mathrm{O}\right)_{3} \mathrm{H}^{+}$. Chem. Phys. Lett. 2000, 321, 225-230. [CrossRef]

19. Marx, D.; Hutter, J. Ab initio molecular dynamics: Theory and implementation. Mod. Methods Algorithms Quantum Chem. 2000, $1,141$.

20. Hohenberg, P.; Kohn, W. Inhomogeneous electron gas. Phys. Rev. 1964, 136, B864. [CrossRef]

21. Kohn, W.; Sham, L.J. Self-consistent equations including exchange and correlation effects. Phys. Rev. 1965, 140, A1133. [CrossRef]

22. Runge, E.; Gross, E.K. Density-functional theory for time-dependent systems. Phys. Rev. Lett. 1984, 52, 997. [CrossRef]

23. Stanton, J.F.; Bartlett, R.J. The equation of motion coupled-cluster method. A systematic biorthogonal approach to molecular excitation energies, transition probabilities, and excited state properties. J. Chem. Phys. 1993, 98, 7029-7039. [CrossRef]

24. Levchenko, S.V.; Krylov, A.I. Equation-of-motion spin-flip coupled-cluster model with single and double substitutions: Theory and application to cyclobutadiene. J. Chem. Phys. 2004, 120, 175-185. [CrossRef] [PubMed]

25. Watts, J.D. An introduction to equation-of-motion and linear-response coupled-cluster methods for electronically excited states of molecules. In Radiation Induced Molecular Phenomena in Nucleic Acids; Springer: Berlin/Heidelberg, Germany, 2008 ; pp. 65-92.

26. Martínez, T.J. Insights for light-driven molecular devices from ab initio multiple spawning excited-state dynamics of organic and biological chromophores. Acc. Chem. Res. 2006, 39, 119-126. [CrossRef]

27. Coe, J.D.; Martínez, T.J. Ab initio molecular dynamics of excited-state intramolecular proton transfer around a three-state conical intersection in malonaldehyde. J. Phys. Chem. A 2006, 110, 618-630. [CrossRef] [PubMed]

28. Coe, J.D.; Levine, B.G.; Martínez, T.J. Ab initio molecular dynamics of excited-state intramolecular proton transfer using multireference perturbation theory. J. Phys. Chem. A 2007, 111, 11302-11310. [CrossRef] 
29. Tao, H.; Levine, B.G.; Martínez, T.J. Ab initio multiple spawning dynamics using multi-state second-order perturbation theory. J. Phys. Chem. A 2009, 113, 13656-13662. [CrossRef]

30. Chakravorty, D.K.; Soudackov, A.V.; Hammes-Schiffer, S. Hybrid quantum/classical molecular dynamics simulations of the proton transfer reactions catalyzed by ketosteroid isomerase: Analysis of hydrogen bonding, conformational motions, and electrostatics. Biochemistry 2009, 48, 10608-10619. [CrossRef]

31. Olsen, S.; Lamothe, K.; Martinez, T.J. Protonic gating of excited-state twisting and charge localization in GFP chromophores: A mechanistic hypothesis for reversible photoswitching. J. Am. Chem. Soc. 2010, 132, 1192-1193. [CrossRef]

32. Hammes-Schiffer, S. Current theoretical challenges in proton-coupled electron transfer: Electron-proton nonadiabaticity, proton relays, and ultrafast dynamics. J. Phys. Chem. Lett. 2011, 2, 1410-1416. [CrossRef]

33. Mashaghi, A.; Partovi-Azar, P.; Jadidi, T.; Anvari, M.; Jand, S.P.; Nafari, N.; Tabar, M.R.R.; Maass, P.; Bakker, H.J.; Bonn, M. Enhanced autoionization of water at phospholipid interfaces. J. Phys. Chem. C 2013, 117, 510-514. [CrossRef]

34. Partovi-Azar, P.; Sebastiani, D. Optimized effective potentials to increase the accuracy of approximate proton transfer energy calculations in the excited state. J. Chem. Phys. 2020, 152, 064101. [CrossRef]

35. Neese, F. The ORCA program system. Wiley Interdiscip. Rev. Comput. Mol. Sci. 2012, 2, 73-78. [CrossRef]

36. Neese, F. Software update: The ORCA program system, version 4.0. Wiley Interdiscip. Rev. Comput. Mol. Sci. 2018, 8, e1327. [CrossRef]

37. Kühne, T.D.; Iannuzzi, M.; Del Ben, M.; Rybkin, V.V.; Seewald, P.; Stein, F.; Laino, T.; Khaliullin, R.Z.; Schütt, O.; Schiffmann, F.; et al. CP2K: An electronic structure and molecular dynamics software package-Quickstep: Efficient and accurate electronic structure calculations. J. Chem. Phys. 2020, 152, 194103. [CrossRef] [PubMed]

38. VandeVondele, J.; Hutter, J. Gaussian basis sets for accurate calculations on molecular systems in gas and condensed phases. J. Chem. Phys. 2007, 127, 114105. [CrossRef] [PubMed]

39. Dunning, T.H., Jr. Gaussian basis sets for use in correlated molecular calculations. I. The atoms boron through neon and hydrogen. J. Chem. Phys. 1989, 90, 1007-1023. [CrossRef]

40. Lin, Y.S.; Li, G.D.; Mao, S.P.; Chai, J.D. Long-range corrected hybrid density functionals with improved dispersion corrections. J. Chem. Theory Comput. 2013, 9, 263-272. [CrossRef] [PubMed]

41. Fetter, A.L.; Walecka, J.D. Quantum Theory of Many-Particle Systems; Courier Corporation: Chelmsford, MA, USA, 2012.

42. Hirata, S.; Head-Gordon, M. Time-dependent density functional theory within the Tamm-Dancoff approximation. Chem. Phys. Lett. 1999, 314, 291-299. [CrossRef]

43. Goedecker, S.; Teter, M.; Hutter, J. Separable dual-space Gaussian pseudopotentials. Phys. Rev. B 1996, 54, 1703. [CrossRef]

44. Krack, M. Pseudopotentials for $\mathrm{H}$ to $\mathrm{Kr}$ optimized for gradient-corrected exchange-correlation functionals. Theor. Chem. Acc. 2005, 114, 145-152. [CrossRef]

45. Car, R.; Parrinello, M. Unified approach for molecular dynamics and density-functional theory. Phys. Rev. Lett. 1985, 55, 2471. [CrossRef]

46. Hutter, J.; Curioni, A. Car-Parrinello molecular dynamics on massively parallel computers. ChemPhysChem 2005, 6, 1788-1793. [CrossRef]

47. Gonze, X.; Amadon, B.; Anglade, P.M.; Beuken, J.M.; Bottin, F.; Boulanger, P.; Bruneval, F.; Caliste, D.; Caracas, R.; Côté, M.; et al. ABINIT: First-principles approach to material and nanosystem properties. Comput. Phys. Commun. 2009, 180, $2582-2615$. [CrossRef]

48. Barzilai, J.; Borwein, J.M. Two-point step size gradient methods. IMA J. Numer. Anal. 1988, 8, 141-148. [CrossRef]

49. Becke, A.D. Density-functional exchange-energy approximation with correct asymptotic behavior. Phys. Rev. A 1988, 38,3098 . [CrossRef] [PubMed]

50. Lee, C.; Yang, W.; Parr, R.G. Development of the Colle-Salvetti correlation-energy formula into a functional of the electron density. Phys. Rev. B 1988, 37, 785. [CrossRef] [PubMed]

51. Grimme, S.; Antony, J.; Ehrlich, S.; Krieg, H. A consistent and accurate ab initio parametrization of density functional dispersion correction (DFT-D) for the 94 elements H-Pu. J. Chem. Phys. 2010, 132, 154104. [CrossRef] [PubMed]

52. Marzari, N.; Vanderbilt, D. Maximally localized generalized Wannier functions for composite energy bands. Phys. Rev. B 1997, 56, 12847. [CrossRef]

53. Martin, R.L. Natural transition orbitals. J. Chem. Phys. 2003, 118, 4775-4777. [CrossRef]

54. Perdew, J.P.; Burke, K.; Ernzerhof, M. Generalized gradient approximation made simple. Phys. Rev. Lett. 1996, 77, 3865. [CrossRef] [PubMed] 